\title{
Überwachung mit Videokameras durch nicht-öffentliche Stellen
}

Am 15. März 2011 hat die Landesbeauftragten für Datenschutz und Informationsfreiheit der Freien Hansestadt Bremen, Imke Sommer, die Orientierungshilfe, Überwachung mit Videokameras und zum Einsatz von Webcams durch nichtöffentliche Stellen' herausgegeben.

Das sind wesentliche Hinweise dieser Dokumentation:

1. Welche Bereiche dürfen überhaupt videoüberwacht werden?

Die Vorschrift des $₫ 6$ b Absatz 1 BDSG regelt nur die Videoüberwachung öffentlich zugänglicher Räume, das sind Räume, die von allen betreten werden dürfen; hierzu zählen unter anderem Kaufhäuser, Museen, Bahnhöfe und Eingangsbereiche von Mehrfamilienhäusern. Videoüberwachung in nicht-öffentlich zugänglichen Räumen, zum Beispiel Privatwohnungen und Privatgrundstücke hat der Gesetzgeber nicht einbezogen. In diesen Fällen ist die Videoüberwachung nur in den von der Rechtsprechung entwickelten Grenzen zulässig. Öffentliche Plätze und Straßen dürfen jedoch nicht von Privaten, sondern nur von der Polizei unter besonderen gesetzlichen Voraussetzungen im Polizeirecht videoüberwacht werden (zum Beispiel $\$ 29$ Bremisches Polizeigesetz).

2. Wer darf unter welchen Voraussetzungen Videoüberwachung einsetzen?

Nur Eigentümerinnen und Eigentümer beziehungsweise sonstige Stellen, die über das Hausrecht verfügen, dürfen nach diesem Gesetz Videoüberwachung einsetzen, wenn es für einen festzulegenden Zweck erforderlich ist und dadurch keine schutzwürdigen Interessen der überwachten Personen überwiegen. Hierzu sind generell folgende Überlegungen vorzunehmen:

- Zwecke und Erforderlichkeit

Bevor eine Videoüberwachung eingesetzt wird, ist zu klären, welches Ziel damit erreicht werden soll, zum Beispiel Verhindern von Einbruch oder Vandalismus. Geprüft werden muss zunächst, ob es nicht die Videoüberwachung vermeidende Mittel gibt, um dieses Ziel zu erreichen; das kann der Einsatz eines Pförtners, Einbau von Sicherheitsschlössern oder einbruchsicheren Türen und Fenstern sein.

Es reicht jedenfalls nicht aus, von vornherein auf die Videoüberwachung zu setzen, ohne vorher Alternativen geprüft zu haben.

- Schutzwürdige Interessen der Betroffenen

Soweit als Ergebnis der Prüfung nur die Videoüberwachung als einzige geeignete Maßnahme in Betracht kommt, muss vor dem Einsatz festgestellt werden, ob und gegebenenfalls welche potentiellen Personengruppen videoüberwacht werden würden und ob deren schutzwürdige Interessen höher zu bewerten sind.

Ein schutzwürdiges Interesse der Betroffenen stellt stets das grundrechtlich garantierte Persönlichkeitsrecht dar. Es umfasst insbesondere das Recht auf Schutz der Privat- und Intimsphäre und das Recht am eigenen Bild.

Schutzwürdige Interessen überwiegen regelmäßig, wenn sensitive Daten erhoben werden (politische Meinungen, religiöse und philosophische Überzeugungen, Gewerkschaftszugehörigkeit, Gesundheit und Sexualität) oder die Intimsphäre verletzt wird. Daher ist die Überwachung von Umkleidekabinen und Toiletten nicht erlaubt. Auch dort, wo die Wahrnehmung von Freiheitsrechten und die Entfaltung der Persönlichkeit von wesentlicher Bedeutung sind, z.B. in Einrichtungen, wo Menschen kommunizieren, essen und trinken oder sich erholen, überwiegen regelmäßig die schutzwürdigen Interessen der Betroffenen.

Die schutzwürdigen Interessen überwiegen grundsätzlich nicht, soweit sich Menschen im Vorraum von Banken mit oder ohne Geldautomaten oder an Zapfsäulen von Tankstellen aufhalten. Der Schutz vor Diebstahl in Kaufhäusern oder das Vermeiden des Besprühens oder Beschmieren von Hausfassaden oder in öffentlichen Verkehrsmitteln kann unter Umständen ebenso gerechtfertigt sein.

Zu klären ist auch, ob die Überwachung dauerhaft oder zeitlich begrenzt (zum Bei- spiel außerhalb von Geschäftszeiten), flächendeckend oder nur punktuell (zum Beispiel an Kellereingängen) erfolgen muss. Bedeutsam ist auch, ob die Videoüberwachung aufgezeichnet werden muss oder ob lediglich ein Monitor zur Beobachtung ausreicht.

- Bestellung einer oder eines betriebli-

chen Datenschutzbeauftragten

Weil die Videoüberwachung besondere Risiken für die Rechte und Freiheiten der überwachten Personen aufweist, muss die verantwortliche Stelle vor dem geplanten Einsatz regelmäßig einen betrieblichen Datenschutzbeauftragten bestellen. Diesem ist die für die Videoüberwachung erstellte Verfahrensbeschreibung nach $\$ 4 \mathrm{e}$ BDSG einschließlich der geplanten technischen und organisatorischen Maßnahmen nach $\$ 9$ BDSG für eine Vorabkontrolle ihrer Zulässigkeit zuzuleiten, damit diese im Rahmen einer Vorabkontrolle prüfen können, ob die erfolgte Rechtsgüterabwägung im Rahmen der vorgenannten materiellrechtlichen Voraussetzungen sowie die technischen und organisatorischen Maßnahmen angemessen sind.

3. Welche Maßnahmen sind vor der Videoüberwachung zu treffen?

Die zu treffenden Maßnahmen hängen auch vom Ergebnis der Vorabkontrolle durch die betrieblichen Datenschutzbeauftragten ab und sind im Einzelnen festzulegen.

- Hinweispflicht

Die Videoüberwachung und die verantwortliche Stelle müssen für Betroffene problemlos erkennbar sein, damit sie frei entscheiden können, ob sie den Bereich betreten wollen.

In der Orientierungshilfe werden darüber hinaus behandelt, Kameraattrappen, wann die Videoaufzeichnungen zu löschen sind und welche Gesichtspunkte beim datenschutzgerechten Einsatz von Webcams wesentlich sind.

Die Orientierungshilfe steht auf der Website der LfDI Bremen unter http:// www.datenschutz-bremen.de/pdf/video_2. $p d f$ zur Verfügung. 